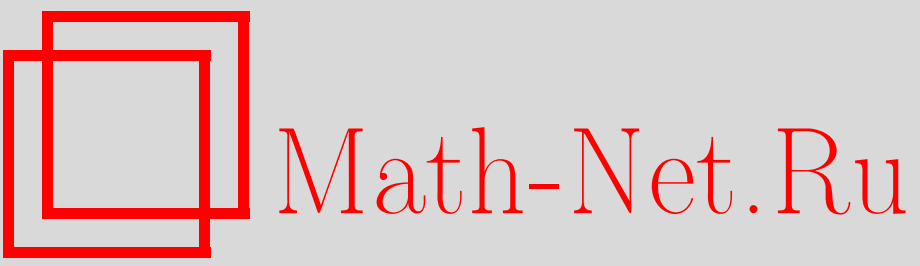

В. М. Гончаренко, О монодромии матричных уравнений Шрёдингера и взаимодействии матричных солитонов, УМН, 2000, том 55, выпуск 5, 175-176

DOI: https://doi.org/10.4213/rm326

Использование Общероссийского математического портала Math-Net.Ru подразумевает, что вы прочитали и согласны с пользовательским соглашением

http://www . mathnet.ru/rus/agreement

Параметры загрузки:

IP: 18.208 .226 .222

26 апреля 2023 г., 08:58:03 


\title{
О МОНОДРОМИИ МАТРИЧНЫХ УРАВНЕНИЙ ШРЕДИНГЕРА И ВЗАИМОДЕЙСТВИИ МАТРИЧНЫХ СОЛИТОНОВ
}

\author{
В. М. ГОНЧАРЕНКО
}

Рассмотрим матричный оператор Шрёдингера $L=-D^{2}+U(x)$ с мероморфным $(d \times d)$-матричнозначным потенциалом $U(x)$. Говорят, что $L$ имеет локальную тривиальную монодромию около регулярной особой точки $x=x_{0} \in \mathbb{C}$, если все решения соответствующего уравнения Шрёдингера $L \psi=\lambda \psi$ однозначны в окрестности $x_{0}$ для любого $\lambda \in \mathbb{C}$. Предположим, что $x_{0}=0$ и разложение Лорана $U(x)$ в окрестности 0 имеет вид

$$
U(x)=\sum_{r \geqslant-2} C_{r} x^{r}
$$

Следующие две теоремы являются обобшением результатов Дюистермаата и Грюнбаума [1], рассматривавших скалярный случай.

Теорема 1 (см. [2]). Матричный оператор Шрёдингера L $c$ потенциалом (1) имеет тривиальную монодромию около $x=0$ тогда и только тогда, когда $C_{-2}=$ $\sum_{j=1}^{k} m_{j}\left(m_{j}-1\right) P_{j}, m_{j} \in \mathbb{N}, 1 \leqslant m_{1}<m_{2}<\cdots<m_{k}=M$, где $P_{j}-$ коммутирующие проекторы на собственные подпространства $V_{j}$, соответствующие собственным значениям $m_{j}\left(m_{j}-1\right): P_{i} P_{j}=\delta_{i j} P_{i}, \sum_{j=1}^{k} P_{j}=I$, и коэффичиенты $C_{l}, l=-1,0, \ldots$, $2 M-3$, удовлетворяют условиям $P_{i} C_{l} P_{j}=0$, если $\left|m_{i}-m_{j}\right| \geqslant l+1$ или $m_{i}+m_{j}=l+3$, $l+5, \ldots, l+2 k+1, \ldots$.

Если потенциал $U(x)$ рациональный и стремится к 0 при $x \rightarrow \infty$, а условия локальной тривиальной монодромии выполнены в каждой из его особенностей $x_{j}, j=1, \ldots, n$, то $U(x)$ имеет вид $\sum_{j=1}^{n} A_{j} /\left(x-x_{j}\right)^{2}$. При этом условия теоремы 1 образуют переопределенную систему на $(d \times d)$-матрицы $A_{j}$ и полюсы $x_{j}$, которая называется уравнениями матричного локуса $[2] . \mathrm{B}$ этом случае мы говорим, что $L$ имеет тривиальную монодромию в $\mathbb{C}$.

Tеорема 2 (см. [2]). Если оператор L имеет тривиальную монодромию в $\mathbb{C}$ и его потенциал рационален и убывает на бесконечности, то L получается матричным преобразованием Дарбу оператора $L_{0}=-D^{2}$, m.е. существует дифференциальньй оператор $A=D^{n}+a_{1}(x) D^{n-1}+\cdots+a_{n}(x)$ c $(d \times d)$-матричными коэффициентами $a_{j}(x)$, сплетающий $L$ и $L_{0}: L A=A L_{0}$.

Другой класс потенциалов операторов Шрёдингера с тривиальной монодромией, которые получаются матричными преобразованиями Дарбу оператора $L_{0}$ - это $N$-солитонные решения $U_{N}(x, t)$ матричного уравнения $\mathrm{K} д \Phi U_{t}=3 U U_{x}+3 U_{x} U-U_{x x x}$ (см. [3]). Чтобы выписать формулу для $U_{N}(x, t)$, мы используем понятие квазидетерминантов, введенное в [4]. Пусть $R=\operatorname{Mat}_{d}(\mathbb{C})$ - алгебра $(d \times d)$-матриц и $X-(N \times N)$-матрица над $R$. Для любых $1 \leqslant i, j \leqslant n$ пусть $r_{i}(X)^{(j)}$ есть $i$-я строка без $j$-го элемента и $c_{j}(X)^{(i)}$ есть $j$-й столбец без $i$-го элемента матрицы $X$. Пусть также $X^{i j}$ - подматрица $X$, полученная удалением $i$-й строки и $j$-го столбца $X$. Тогда по определению квазидетерминант $|X|_{i j}=x_{i j}-r_{i}(X)^{(j)}\left(X^{i j}\right)^{-1} c_{j}(X)^{(i)}$.

Пусть теперь $A$ есть $(N \times N)$-матрица, $(i, j)$-м элементом которой является $(d \times d)$-матрица $A_{i j}=\delta_{i j} I+R_{j}(t) e^{-\left(\lambda_{i}+\lambda_{j}\right) x} /\left(\lambda_{i}+\lambda_{j}\right)$, где $R_{j}(t)=R_{j} e^{8 \lambda_{j}^{3} t}$ (cp. [5]) с некоторыми $(d \times d)$-матрицами $R_{j}$ и $0<\lambda_{1}<\cdots<\lambda_{N}$. Тогда $U_{N}(x, t)=-2\left(\sum_{j=1}^{N}\left|A^{(j)}\right|_{j j}|A|_{j j}^{-1}\right)^{\prime}$, где $A^{(j)}$ получается из $A$ дифференцированием $j$-й строки (ср. [6]).

Далее мы будем предполагать, что $R_{j}=p_{j} \otimes q_{j}, j=1, \ldots, N$, где $p_{j}-d$-вектор, $q_{j}$ - ковектор, а $\left(p_{j}, q_{j}\right) \neq 0$ - их естественное спаривание. Тогда односолитонное решение матричного уравнения $\mathrm{K} д \Phi$ имеет вид

$$
U_{1}(x, t)=-\frac{2 \lambda_{1}^{2} p_{1} \otimes q_{1}}{\left(p_{1}, q_{1}\right)} \operatorname{sech}^{2} \lambda_{1}\left(\theta_{1}-\phi_{1}\right), \quad \phi_{1}=\frac{1}{2 \lambda_{1}} \ln \frac{\left(p_{1}, q_{1}\right)}{2 \lambda_{1}},
$$

Работа выполнена при поддержке университета Лафбборо и гранта INTAS № 96-0770. 
где $\theta_{1}=x-4 \lambda_{1}^{2} t$. Мы назьваем $\frac{2 \lambda_{1}^{2} p_{1} \otimes q_{1}}{\left(p_{1}, q_{1}\right)}=2 \lambda_{1}^{2} \frac{R_{1}}{\operatorname{tr} R_{1}}$ матричной амплитудой (поляризацией) солитона и $\phi_{1}-$ его фазой.

Рассмотрим теперь 2-солитонное решение $U_{2}$. Заметим, что оно регулярно, если $\left(p_{1}, q_{1}\right)>0$, $\left(p_{2}, q_{2}\right)>0$ и $\alpha>0$, где $\alpha=1-4 \lambda_{1} \lambda_{2}\left(p_{1}, q_{2}\right)\left(p_{2}, q_{1}\right) /\left(\left(\lambda_{1}+\lambda_{2}\right)^{2}\left(p_{1}, q_{1}\right)\left(p_{2}, q_{2}\right)\right)$. С точностью до экспоненциально убывающих при $|t| \rightarrow \infty$ членов мы имеем: при $t \rightarrow-\infty$

$$
U_{2}(x, t) \sim-\frac{2 \lambda_{1}^{2} p_{1} \otimes q_{1}}{\left(p_{1}, q_{1}\right)} \operatorname{sech}^{2} \lambda_{1}\left(\theta_{1}-\phi_{1}^{-}\right)-\frac{2 \lambda_{2}^{2} \hat{p}_{2} \otimes \hat{q}_{2}}{\left(\hat{p}_{2}, \hat{q}_{2}\right)} \operatorname{sech}^{2} \lambda_{2}\left(\theta_{2}-\Phi_{2}^{-}\right),
$$

где $\theta_{j}=x-4 \lambda_{j}^{2} t, \phi_{1}^{-}=\frac{1}{2 \lambda_{1}} \ln \frac{\left(p_{1}, q_{1}\right)}{2 \lambda_{1}}, \phi_{2}^{-}=\frac{1}{2 \lambda_{2}} \ln \frac{\left(\hat{p}_{2}, \hat{q}_{2}\right)}{2 \lambda_{2}\left(p_{1}, q_{1}\right)^{2}}$ и $\hat{p}_{2}=\left(p_{1}, q_{1}\right) p_{2}-$ $\frac{2 \lambda_{1}}{\lambda_{1}+\lambda_{2}}\left(p_{2}, q_{1}\right) p_{1}, \hat{q}_{2}=\left(p_{1}, q_{1}\right) q_{2}-\frac{2 \lambda_{1}}{\lambda_{1}+\lambda_{2}}\left(p_{1}, q_{2}\right) q_{1}$.

При $t \rightarrow+\infty$

$$
U_{2}(x, t) \sim-\frac{2 \lambda_{1}^{2} \hat{p}_{1} \otimes \hat{q}_{1}}{\left(\hat{p}_{1}, \hat{q}_{1}\right)} \operatorname{sech}^{2} \lambda_{1}\left(\theta_{1}-\phi_{1}^{+}\right)-\frac{2 \lambda_{2}^{2} p_{2} \otimes q_{2}}{\left(p_{2}, q_{2}\right)} \operatorname{sech}^{2} \lambda_{2}\left(\theta_{2}-\phi_{2}^{+}\right),
$$

где $\phi_{1}^{+}=\frac{1}{2 \lambda_{1}} \ln \frac{\left(\hat{p}_{1}, \hat{q}_{1}\right)}{2 \lambda_{1}\left(p_{2}, q_{2}\right)^{2}}, \phi_{2}^{+}=\frac{1}{2 \lambda_{2}} \ln \frac{\left(p_{2}, q_{2}\right)}{2 \lambda_{2}}$ и $\hat{p}_{1}=\left(p_{2}, q_{2}\right) p_{1}-\frac{2 \lambda_{2}}{\lambda_{1}+\lambda_{2}}\left(p_{1}, q_{2}\right) p_{2}, \hat{q}_{1}=$ $\left(p_{2}, q_{2}\right) q_{1}-\frac{2 \lambda_{2}}{\lambda_{1}+\lambda_{2}}\left(p_{2}, q_{1}\right) q_{2}$. Поэтому $U_{2}(x, t)$ описывает взаимодействие двух 1-солитонов со скоростями $4 \lambda_{j}^{2}, j=1,2$. Сдига фаз $\Delta_{j}=\phi_{j}^{+}-\phi_{j}^{-}$имеют вид $\Delta_{1}=\frac{1}{2 \lambda_{1}} \ln \alpha, \Delta_{2}=-\frac{1}{2 \lambda_{2}} \ln \alpha$.

Заметим, что $\Delta_{1}$ может принимать как положительные, так и отрицательные значения, т.е. в матричном случае медленный солитон после взаимодействия может сдвигаться не только назад, как в скалярном случае (см. [5]), но и вперед, если $\alpha>1$, т.е. $\left(p_{1}, q_{2}\right)\left(p_{2}, q_{1}\right)<0$.

Другой замечательной особенностью взаимодействия солитонов является изменение матричной амплитуды (ср. [7]): как легко видеть из формул, приведенных выше, амплитуда первого солитона меняется $2 \lambda_{1}^{2} R_{1} / \operatorname{tr} R_{1} \rightarrow 2 \lambda_{1}^{2} \widehat{R}_{1} / \operatorname{tr} \widehat{R}_{1}$ и второго $-2 \lambda_{2}^{2} \widehat{R}_{2} / \operatorname{tr} \widehat{R}_{2} \rightarrow 2 \lambda_{2}^{2} R_{2} / \operatorname{tr} R_{2}$, где $\widehat{R}_{1}=\hat{p}_{1} \otimes \hat{q}_{1}$ и $\widehat{R}_{2}=\hat{p}_{2} \otimes \hat{q}_{2}$.

В $N$-солитонном случае использование стандартных аргументов (см., например, [8]), основанных на существовании потоков, коммутируюших с потоком матричного уравнения Кд $\Phi$ [9], позволяет доказать следующий результат, хорошо известный в скалярном случае.

Теорема 3. Для взаимодействия $N$ солитонов матричного уравнения КдФ полный сдвиг фаз и изменение амплитуд солитонов могут быть вычислены как результат их попарного взаимодействия, описанного выше. В частности, результат взаимодействий не зависит от их порядка и многочастичный эффект отсутствует.

Автор хотел бы выразить свою искреннюю признательность профессору А.П. Веселову за постановку задачи и многочисленные обсуждения.

\section{СПИСОК ЛИТЕРАТУРЫ}

[1] Duistermaat J. J., Grünbaum F. A . // Comm. Math. Phys. 1986. V. 103. P. 177-240. [2] Goncharenko V. M., Veselov A. P. // J. Phys. A. 1998. V. 31. P. 5315-5326. [3] Lax P. D. // Comm. Pure Appl. Math. 1968. V. 21. P. 467-490. [4] Гельфанд И. М., Ретах В. С. // Функц. анализ и его прил. 1991. Т. 25. № 2. С. 13-25. [5] Захаров В. Е., Манаков С. В., Новиков С.П., Питаевский Л. В. Теория солитонов. Метод обратной задачи. М.: Наука, 1980. [6] Etingof P., Gelfand I., Retakh V. Factorization of differential operators, quasideterminants, and nonabelian Toda field equation // q-alg/971008. [7] Calogero F., Degasperis A. // Nuovo Cimento B (1). 1977. V. 39. P. 1-53. [8] Ньюэлл А. Солитоны в математике и физике. М.: Мир, 1989. [9] Olmedilla E., Martinez Alonso L., Guil F. // Nuovo Cimento B (1). 1981. V. 61. P. 49-61. 